\title{
Estudio del comportamiento de los campos electromagnéticos en la banda de $1900 \mathrm{MHz}$
}

\author{
Nataly Idrovo López / Franklin Chenche Villacís
}

\section{RESUMEN}

Las comunicaciones inalámbricas son de uso cotidiano por la gran variedad de aplicaciones de comunicación, entretenimiento, educación y control que permiten una mayor conectividad y movilidad al usuario final para acceder a estos servicios. Esto ha ocasionado que las personas estén expuestas a campos electromagnéticos generados por equipos creados por el hombre de manera continua. Por esta razón muchas organizaciones han visto la necesidad de efectuar estudios y posteriormente generar recomendaciones referentes al control de estos campos. El desarrollo de este artículo está centrado en la aplicación de varios conceptos presentados en estas recomendaciones para analizar el comportamiento de los campos electromagnéticos correspondientes a un segmento del espectro, generados por el servicio de telefonía móvil, en función de la distancia de la fuente. Para esto fue necesario realizar un análisis del sitio de medición para determinar la presencia de señales externas interferentes en la banda de $1900 \mathrm{MHz}$, así como mediciones de los niveles de potencia de la fuente en diferentes puntos, para finalmente realizar mediciones de intensidad de campo eléctrico de uno a diez metros de la fuente radiante y poder evidenciar que estos niveles disminuyen de manera exponencial conforme aumenta la distancia de la fuente.

\section{PALABRAS CLAVE}

Campos electromagnéticos, telefonía móvil, intensidad de campo eléctrico, región de campo lejano, región de campo cercano.

\section{ABSTRACT}

Wireless communications are in daily use because of the wide variety of communication, entertainment, education, and control applications they allow they allow a major connectivity and mobility for the final user to access for the services. This has meant that people are exposed to electromagnetic fields generated by man-made equipment continuously. For this reason many organizations have seen the need to carry out studies and later generate recommendations concerning the control of these fields. The development of this article focuses on the application of various concepts presented in these recommendations to analyze the behavior of electromagnetic fields of a segment of the spectrum, generated by mobile phone service, depending on the distance from the source. This required an analysis of the measurement site to determine the presence of interfering external signals in the band of $1900 \mathrm{MHz}$, and measurements of the power levels of the source at different points, to finally perform measurements of electric field strength from one to ten meters from the source and show that these levels decrease with increasing distance from it.

\section{REYWIORDS}

Electromagnetic fields, mobile telephony, electric field intensity, far-field region, near-field region. 


\section{Introducción}

En el mundo actual (2014), las comunicaciones inalámbricas se han ruelto una parte esencial de la vida de cada persona, principalmente por la gran variedad de ventajas que trae consigo. Inevitablemente, esto ha ocasionado que todos estén expuestos a campos electromagnéticos, ya sean estos de frecuencias muy bajas, como los provenientes de aparatos eléctricos $y$ líneas de energía, o de radiofrecuencia, como los originados por las estaciones de radio y televisión, antenas celulares y teléfonos inalámbricos (BioInitiative Working Group, 2012, pág. 3).

Esta continua exposición a campos electromagnéticos ha llevado a varias instituciones nacionales e internacionales, como la Unión Internacional de Telecomunicaciones (UIT) y el Consejo Nacional de Telecomunicaciones (CONATEL), a desarrollar recomendaciones y procedimientos de control para asegurar el cumplimiento de los límites de referencia establecidos y así disminuir la exposición de seres vivos a altos niveles de intensidad de campos electromagnéticos.

Adicionalmente, se han llevado a cabo un sinnúmero de estudios en los que se emplean procedimientos, tanto prácticos como teóricos, para identificar el comportamiento de estos campos en diferentes circunstancias.

Un ejemplo de esto es el caso de estudio elaborado por un departamento del Gobierno de Canadá en el que se efectúan mediciones de la exposición de radiofrecuencia de dispositivos Wi-Fi (Industry Canada, 2012). Otro escrito relevante es el generado en el Departamento de Ingeniería Eléctrica de la Universidad del País Vasco, titulado Análisis del campo magnético en una línea eléctrica de $A T$, en el cual sus autores realizan un análisis de los campos magnéticos presentes en una línea eléctrica de alta tensión (Moreno, Güemes, \& Pico, s.f.). Por otro lado, un artículo publicado en la revista Romanian Journal of P/jysics, realiza una comparación de los valores de densidad de potencia estimados y medidos provenientes de estaciones base celulares (Miclaus \& Bechet, 2007).

El principal objetivo de este trabajo es analizar el comportamiento de los campos electromagnéticos a medida que aumenta la distancia del receptor respecto a la fuente, aplicando conceptos de las metodologías utilizadas en algunos de los trabajos mencionados. Para realizar este estudio se llevarán a cabo mediciones de intensidad de campo eléctrico de uno a diez metros a partir de la fuente para así observar si estos niveles disminuyen conforme aumenta la distancia.

Para el cumplimiento de este objetivo será necesario analizar el comportamiento espectral de la fuente y realizar un monitoreo del sitio donde se efectuarán las mediciones para determinar si existen campos electromagnéticos externos que puedan afectar los resultados.

\section{Fundamentación teórica}

\section{Ondas electromagnéticas}

"Las ondas de radio son un tipo de energía electromagnética (o radiación electromagnética - EMR), formada por campos eléctricos y magnéticos desplazándose juntos a través del espacio" (GSMA, 2014, pág. 2). Estas ondas no necesitan un medio material para propagarse; lo hacen a partir de una carga eléctrica oscilante en forma de campos eléctricos y magnéticos. Los campos eléctricos se originan en diferencias de voltaje, mientras que los campos magnéticos tienen su origen en corrientes eléctricas (Organización Mundial de la Salud, s.f.).

Las ondas electromagnéticas se clasifican en términos de frecuencia y de longitud de onda. La frecuencia representa el número de ciclos por segundo, y la longitud de onda 
se refiere a la distancia en metros entre dos puntos continuos de una onda periódica, la cual es inversamente proporcional a la frecuencia y está dada por la siguiente ecuación:
Donde:
$\lambda$ : Longitud de onda (m)
$\mathrm{V}$ : Velocidad $(\mathrm{m} / \mathrm{s})$
f: frecuencia $(\mathrm{Hz})$

espectro está dividido en secciones basadas en la longitud de onda de las señales. En orden creciente de frecuencias, o decreciente de longitudes de onda, el espectro electromagnético está compuesto por: ondas estáticas, ondas de radio, microondas, radiación infrarroja, luz visible, radiación ultravioleta, rayos $\mathrm{X}$ y rayos gamma, como se muestra en la Figura 1.

En el espacio libre, $\mathrm{V}$ equivale a $3 \times 10^{8} \mathrm{~m} / \mathrm{s}$, el valor de la velocidad de la luz (Consejo Nacional de Telecomunicaciones, 2005, pág. 7).

Los campos electromagnéticos se determinan por la intensidad de campo eléctrico (E) e intensidad de campo magnético (H). El Regla-

Figura \# 1

Gráfico del espectro radioeléctrico e ilustraciones de los principales servicios que funcionan en cada banda de frecuencias.
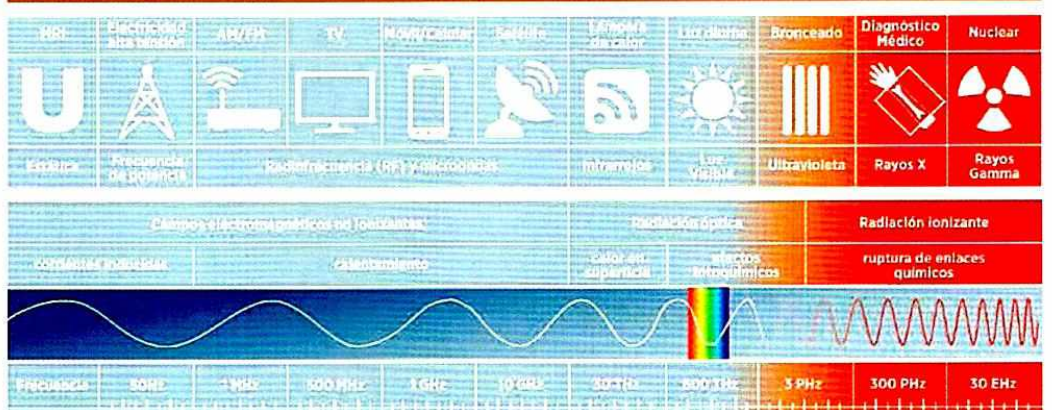

MRI = Magnetic resonance imaging |lmagen por resonancia magnétical; $A M / F M=$ Amplitud modulada/Frecuencia modulada; TV = Televisión. Tomado de "Comunicaciones móviles y salud", por GSM Association, 2014 disponible en http://www.gsma.com/publicpolicy/mobile-communications-and-health mento de protección de emisiones de radiación no ionizante generadas por uso de frecuencias del espectro radioeléctrico define la intensidad de campo eléctrico como la "fuerza por unidad de carga que experimenta una partícula cargada dentro de un campo eléctrico" (Consejo Nacional de Telecomunicaciones, 2005, pág. 7), la cual es expresada en voltios por metro $(\mathrm{V} / \mathrm{m})$. Mientras que el mismo documento define la intensidad de campo magnético como la "magnitud vectorial axial que junto con la inducción magnética, determina un campo magnético en cualquier punto del espacio" (Consejo Nacional de Telecomunicaciones, 2005, pág. 7) y se mide en amperios por metro $(\mathrm{A} / \mathrm{m})$.

\section{Espectro electromagnético}

Como se mencionó anteriormente, las ondas electromagnéticas pueden tener frecuencias muy variadas. A esta distribución de frecuencias o de longitud de onda, se le conoce como espectro electromagnético. El
Para el propósito de este estudio, se prestará especial atención a la sección del espectro correspondiente a las ondas de radio y a las microondas, específicamente aquellas en las que opera la telefonía móvil. Las ondas de radio o radiofrecuencia se refieren a aquellas frecuencias entre los $300 \mathrm{~Hz}$ y los $300 \mathrm{GHz}$, y las microondas, comprendidas en la sección anterior, incluyen el rango de frecuencias entre $300 \mathrm{MHz}$ y $300 \mathrm{GHz}$ (Comisión Internacional para la Protección de las Radiaciones no Ionizantes, 1998).

\section{Normativa y recomendaciones nacio- nales e internacionales}

Existe un sinnúmero de recomendaciones, procedimientos y documentos de carácter legal, tanto en el Ecuador como en el resto del mundo referentes a las emisiones electromagnéticas. A continuación se describe el contenido de tres de estos documentos que son de especial importancia en el país. 
Recomendaciones para limitar la exposición a campos eléctricos, magnéticos y electromagnéticos (hasta $300 \mathrm{GHz}$ )

Este documento fue emitido por la Comisión Internacional para la Protección de Radiaciones no Ionizantes en el año 1998, con el objetivo principal de establecer restricciones básicas y niveles de referencia respecto a la exposición a campos electromagnéticos. Los límites que establece esta Comisión incluyen funciones como investigar los peligros asociados con los campos electromagnéticos, desarrollar recomendaciones internacionales, y tratar aspectos sobre protección contra este tipo de emisiones (Comisión Internacional para la Protección de las Radiaciones no Ionizantes, 1998).

Recomendación UIT-T K.52: Orientación sobre el cumplimiento de los límites de exposición de las personas a los campos electromagnéticos

Esta Recomendación, publicada en el año 2000 por la Unión Internacional de Telecomunicaciones, tiene como objetivo proporcionar guías que permitan el cumplimiento de los niveles de referencia para los campos electromagnéticos en el rango de frecuencias de $9 \mathrm{kHz}$ y $300 \mathrm{GHz}$ (Unión Internacional de Telecomunicaciones, 2000).

La Recomendación UIT-T K.52 proporciona métodos para estimar la densidad de potencia en el campo lejano a una distancia $R$ de la fuente, utilizando la siguiente ecuación simplificada (Unión Internacional de Telecomunicaciones, 2000, pág. 26):

$$
S=\frac{P G_{i}}{4 \pi R^{2}}=\frac{P I R E}{4 \pi R^{2}}
$$

\section{Donde:}

S: densidad de potencia $(\mathrm{W} / \mathrm{m} 2)$

P: potencia suministrada a la fuente de radiación (W)

Gi: factor de ganancia de la fuente de ra- diación con respecto a un radiador isótropo $(\mathrm{dBi})$

R: distancia desde la fuente de radiación (m)

PIRE: potencia isotrópica radiada equivalente de la antena (W)

Para estimar la exposición a nivel del suelo de una antena de altura $\mathrm{h}[\mathrm{m}]$, con haz de inclinación $\theta$ a una distancia $\mathrm{x}[\mathrm{m}]$ de la torre, como se observa en la Figura 2, se debe utilizar las siguientes expresiones (Unión Internacional de Telecomunicaciones, 2000, pág. 26):

$$
\begin{aligned}
& R^{2}=h^{\prime 2}+x^{2} \\
& \theta=\tan ^{-1}\left(\frac{h^{\prime}}{x}\right)
\end{aligned}
$$

Donde h' $[\mathrm{m}]$ es igual a h menos la altura del punto de evaluación.

\section{Figura \#2}

Diagrama de exposición al nivel del suelo debido a una antena elevada.

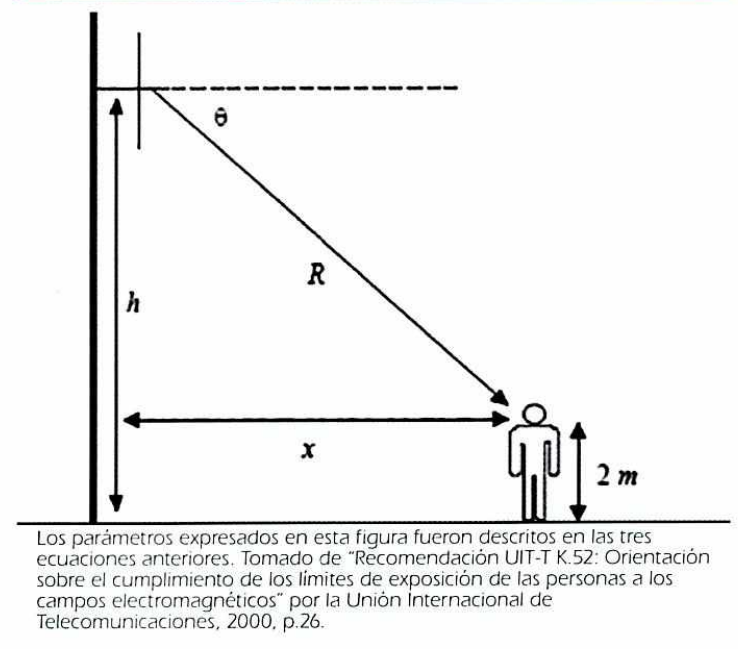

Reglamento de protección de emisiones de radiación no ionizante generadas por uso de frecuencias del espectro radioeléctrico

Este Reglamento fue emitido por el Consejo Nacional de Telecomunicaciones mediante Resolución 01-01-CONATEL-2005 en enero de 2005. Entre los objetivos prin- 
cipales se encuentra establecer los límites de protección, basados en los propuestos en el documento Recomendaciones para limitar la exposición a campos eléctricos, magnéticos y' electromagnéticos (basta $300 \mathrm{GH}$ ) (Comisión Internacional para la Protección de las Radiaciones no Ionizantes, 1998). Adicionalmente, establece un procedimiento para el monitoreo y control de los campos electromagnéticos.

Este documento está basado en su mayoría en la Recomendación UIT-T K.52 ya que hace referencia a las tres zonas existentes alrededor de una estación base, indica cómo determinar la distancia de seguridad conociendo la densidad de potencia en un punto $x$ de la antena, entre ottas cosas.

\section{Metodología}

El diseño del presente estudio es exploratorio documental y cualitativo debido a que la información necesaria para el desarrollo del mismo se la obtuvo a través de la revisión de fuentes bibliográficas y observación de los resultados conseguidos por medio de la realización de mediciones de intensidad de campo eléctrico.

Las pruebas consisten en la simulación de una señal celular utilizando un equipo inhibidor que genere una señal en las bandas de frecuencias concesionadas a las operadoras de telefonía móvil en el país para medir la intensidad de campo eléctrico existente en varios puntos a partir de la fuente y comprobar la variación en función de la distancia, de uno a diez metros de distancia desde el equipo generador de señal hasta el punto de medición. Las pruebas fueron divididas en cuatro fases: la primera consistió en el análisis de los campos electromagnéticos existentes en el sitio de medición para determinar la presencia de señales externas en las diferentes bandas de frecuencia, la segunda fase involucró mediciones de los niveles de potencia de recepción del equipo inhibidor en diez puntos, luego se realizaron mediciones de intensidad de campo eléctrico en diez puntos sin la presencia de una fuente, y finalmente, la última fase consistió en las mediciones de intensidad de campo eléctrico utilizando el inhibidor como generador de señales.

Las mediciones fueron realizadas entre el 20 y 28 de marzo de 2014. El área en la que se llevaron a cabo las pruebas tiene una dimensión de 16.7 por 6.3 metros y mantenía una temperatura constante de $22^{\circ} \mathrm{c}$. Se seleccionó este sitio debido a la inmunidad electromagnética que presentaba frente a señales de ciertas operadoras de telefonía móvil lo que permitió simular un ambiente de laboratorio y que factores externos no afecten los resultados de las pruebas de manera significativa.

En primer lugar, se realizó un monitoreo del comportamiento espectral en el rango de frecuencias de $88 \mathrm{MHz}$ a $4 \mathrm{GHz}$ en el interior del laboratorio con el fin de conocer las señales presentes en el mismo y poder concluir si el área establecida para la realización de las pruebas se encuentra lejos de la influencia de campos electromagnéticos generados por fuentes externas. Los equipos utilizados para estas mediciones fueron un analizador de espectro portátil Anritsu MS2724B Spectrum Master de $9 \mathrm{kHz}$ a $20 \mathrm{GHz}$ y una antena de tipo logarítmica periódica Aaronia Hyperlog de 0.38 a $18 \mathrm{GHz}$. Las mediciones fueron realizadas en el punto indicado en la Figura 3 , el cual fue seleccionado ya que luego de realizar un barrido general del sitio se determinó que en esta posición se encontraron campos electromagnéticos de mayor intensidad, y se debía garantizar la inmunidad del área incluso en el punto con más influencia de señales electromagnéticas. En la Figura 3 se muestra un bosquejo del área donde se realizaron las mediciones. 
Figura \#3

Diagrama de exposición al nivel del suelo debido a una antena elevada.

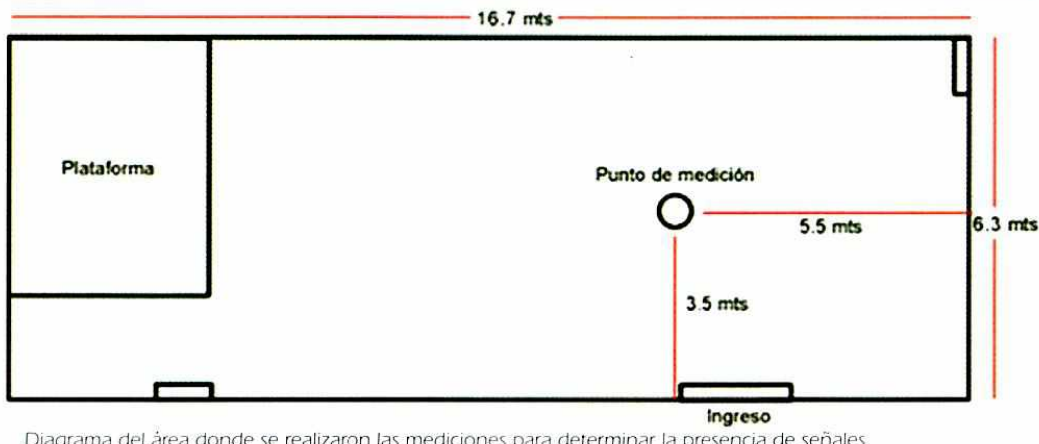

Diagrama del área donde se realizaron las mediciones para determinar la presencia de señales. Fuente: Elaboración propia.

Las mediciones fueron realizadas en frecuencias comprendidas entre $88 \mathrm{MHz}$ y 4 $\mathrm{GHz}$, detectando portadoras en las bandas

\section{Figura \#4}

Gráfica espectral del rango de frecuencias de $800 \mathrm{MHz}$ a $980 \mathrm{MHz}$.

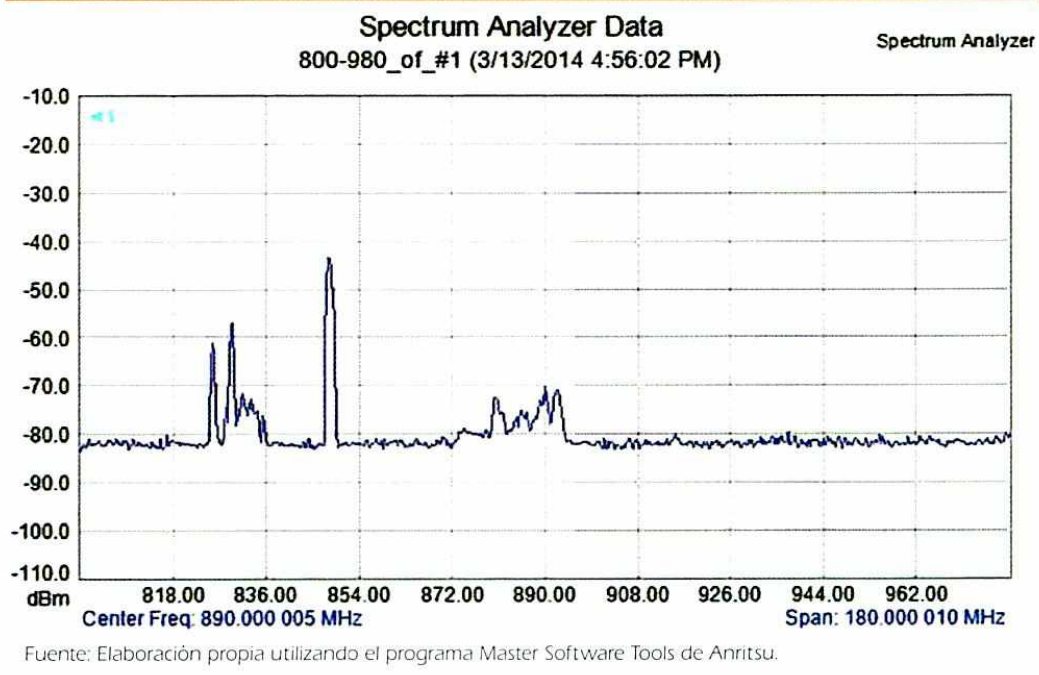

\section{Figura \#5}

Gráfica espectral del rango de frecuencias de $1000 \mathrm{MHz}$ a $2000 \mathrm{MHz}$.

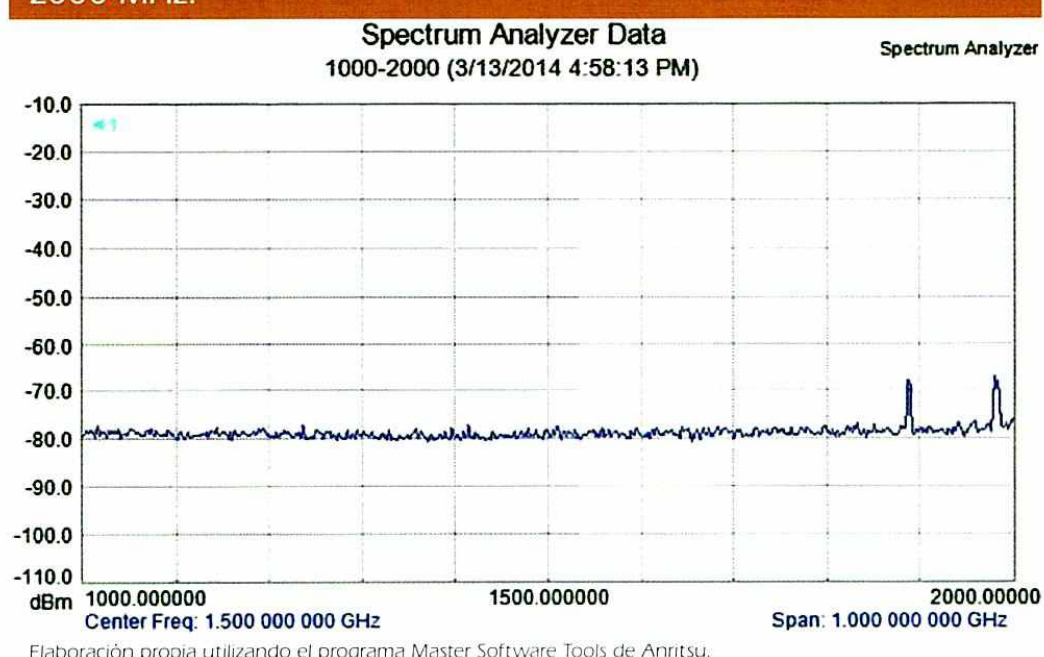

de radiodifusión FM, televisión abierta analógica y digital y Servicio Móvil Avanzado. Para el propósito de este estudio se analizaron tan sólo los gráficos de 800 a $980 \mathrm{MHz}$ (ver Figura 4) y 1000 a $2000 \mathrm{MHz}$ (ver Figura 5) ya que en estos rangos están contenidas las bandas correspondientes al servicio de telefonía móvil en el país.

A partir de los valores obtenidos en estas dos gráficas se puede determinar que existen portadoras de telefonía móvil correspondientes a las operadoras CONECEL S.A. (Claro) y OTECEL S.A. (Movistar) en la banda de $850 \mathrm{MHz}$ y de las operadoras CONECEL S.A. y CNT E.P. en la de $1900 \mathrm{MHz}$.

Para la selección del rango de frecuencias objeto de estudio, se tomó en cuenta que la antena sectorial y el equipo inhibidor tienen la facultad de trabajar en la banda de 1900 MHz. Debido a que no existen señales, tanto de transmisión como de recepción en las bandas concesionadas a la operadora OTECEL S.A., de acuerdo a lo establecido en el Contrato de Concesión de esta operadora (ver Tabla 1), 


\begin{tabular}{|c|c|c|c|c|}
\hline \multicolumn{5}{|c|}{$\begin{array}{l}\text { Bandas de frecuencias esenciales asignadas a la } \\
\text { operadora OTECEL S.A. }\end{array}$} \\
\hline Rango & Bandas & Limite Inferior (MHz) & Limite Superior (MHz) & $\mathrm{AB}(\mathrm{MHz})$ \\
\hline \multirow{4}{*}{$850 \mathrm{MHz}$} & B1 & 835 & 845 & 10 \\
\hline & $\mathrm{BI}^{\prime}$ & 880 & 890 & 10 \\
\hline & B2 & 846.5 & 849 & 2.5 \\
\hline & $B 2^{\prime}$ & 891.5 & 894 & 2.5 \\
\hline \multirow{3}{*}{$1900 \mathrm{MHz}$} & D & 1865 & 1870 & 5 \\
\hline & $D^{\prime}$ & 1945 & 1950 & 5 \\
\hline & & & TOTAL & 35 \\
\hline
\end{tabular}

se seleccionó la banda $\mathrm{D}^{\prime}$, que incluye las frecuencias entre 1945 y $1950 \mathrm{MHz}$, para la realización de las pruebas que comprende la presente investigación (SENATEL y OTECEL S.A., 2008).

La segunda etapa del procedimiento incluye el estudio del comportamiento del equipo inhibidor Phone Jammer PJ004, para lo cual se realizaron mediciones de la potencia de recepción en cada uno de los diez puntos de medición a una altura de 1.5 metros; la ubicación de estos puntos se puede observar en la Figura 6. Para realizar estas mediciones se utilizó una antena sectorial Kathrein de tipo panel número 742212 (ver Figura 7), conectada al generador de señales mediante un cable con terminales SMA-N y colocada en un trípode no conductivo marca Berlebach, alcanzando una altura máxima de 2.23 metros. Para efectuar estas mediciones se utilizó el analizador de espectro Anritsu MS2724B Spectrum Master empleado en las pruebas anteriores y una antena omnidireccional de aproximadamente 3 $\mathrm{cm}$ de longitud (ver Figura 8 ).
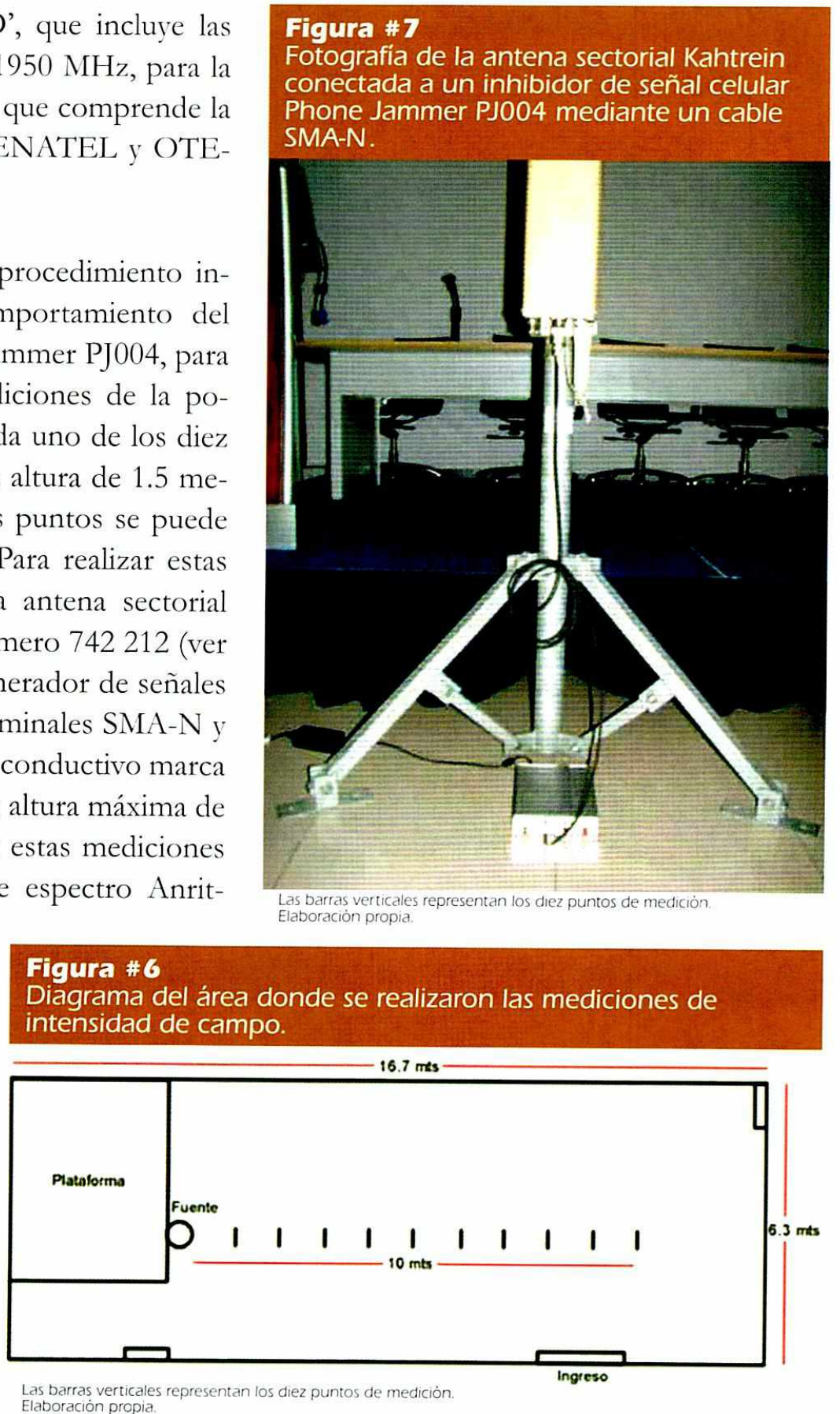
Los resultados de estas mediciones se muestran en las Figuras 9, 10 y 11 en las que se puede observar la disminución de la potencia de recepción conforme va aumentando la distancia de la fuente, resultando en una diferencia de aproximadamente $12 \mathrm{dBm}$ entre el primer y el último punto, como se observa en la Figura 11.
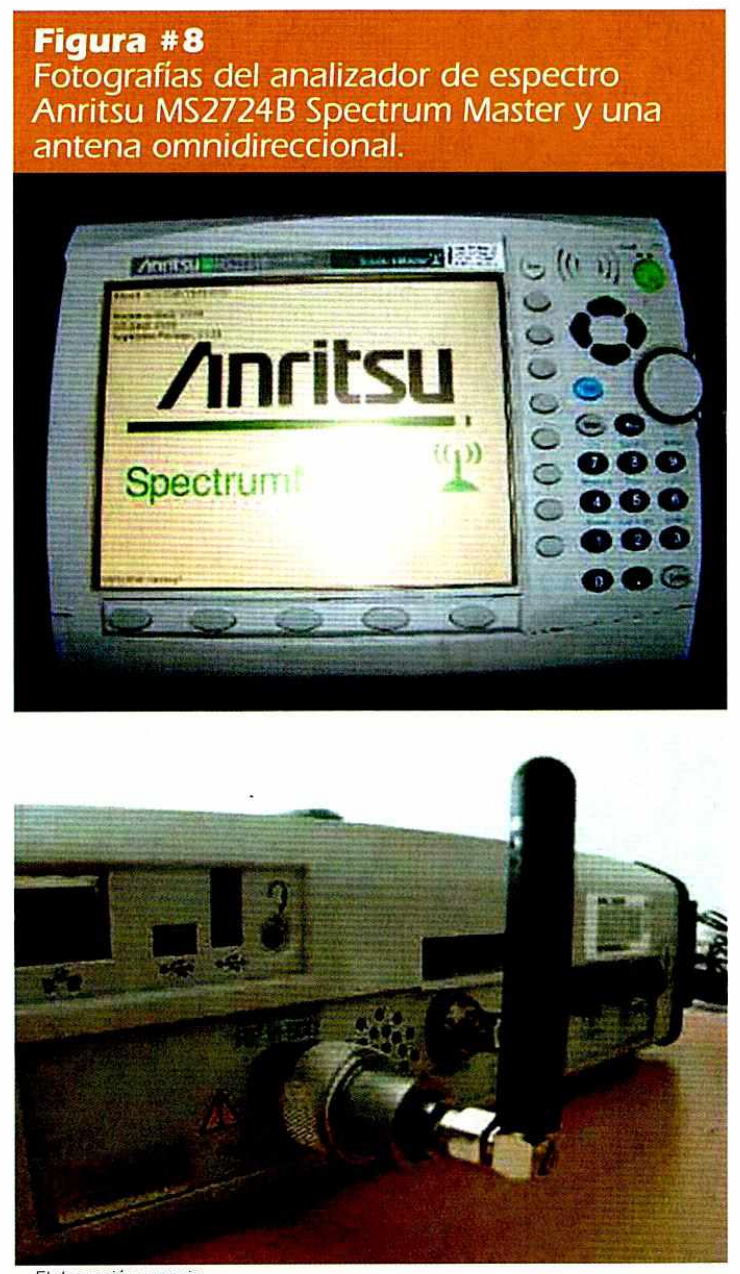

Elaboración propia.

\section{Figura \#9}

Gráfica espectral de las mediciones de potencia de recepción a

uno, dos, tres, cuatro y cinco metros de la fuente.

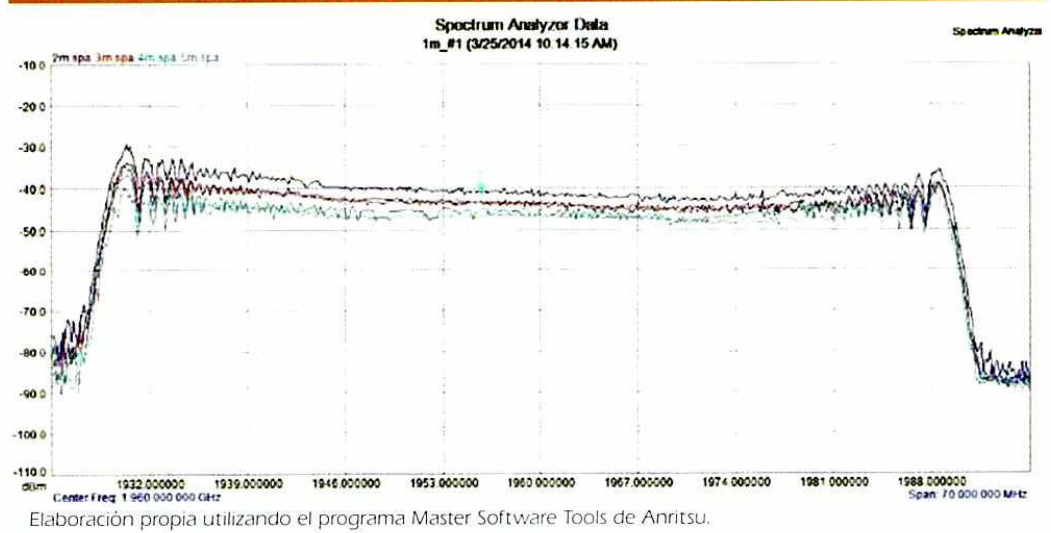

En las Figuras 9 y 10 se observa la disminución progresiva de la potencia conforme el receptor se va alejando de la fuente. En la Figura 11 se puede evidenciar claramente la diferencia de aproximadamente $12 \mathrm{dBm}$ entre la medición realizada en el primer punto y en el último. Adicionalmente, se observa que la frecuencia central de estas señales es $1960 \mathrm{MHz}$ y tienen un ancho de banda de aproximadamente $60 \mathrm{MHz}$

Para las siguientes pruebas fue necesario el uso de un medidor de radiación y una sonda isotrópica que permita capturar los valores de intensidad de campo eléctrico actuales durante 30 minutos e ir almacenándolos para su posterior análisis.

El medidor de radiación utilizado es el Narda SRM-3000, el cual es un medidor de banda angosta o selectivo en frecuencia. Mientras que los dispositivos de banda ancha permiten conocer la intensidad de campo de todas las frecuencias combinadas en un punto o área, la ventaja de los dispositivos de banda angosta es que permiten identificar el nivel de intensidad de campo producido por cada una de las fuentes presentes para así conocer la contribución de cada una de ellas por separado al nivel de emisión en un punto determinado (Skvarca \& Aguirre, 2006, pág. 210).

El Narda SRM-3000 permite medir campos electromagnéticos de frecuencias desde 75 $\mathrm{MHz}$ hasta $3 \mathrm{GHz}$ gracias a una sonda isotrópica tri-axial conectada al dispositivo. La ventaja de este tipo de antenas es que hace posible detectar los tres componentes espaciales automáticamente $\sin$ tener que medir 
Figura \# 10

Gráfica espectral de las mediciones de potencia de recepción a

seis, siete, ocho, nueve y diez metros de la fuente.

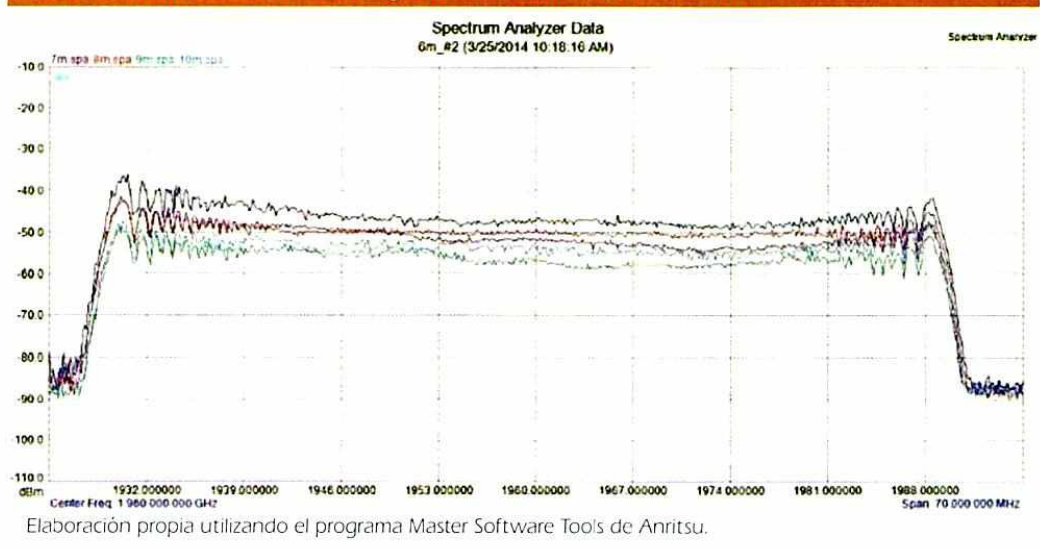

\section{Figura \# 11}

Gráfica espectral de las mediciones de potencia de recepción a uno y diez metros de la fuente.

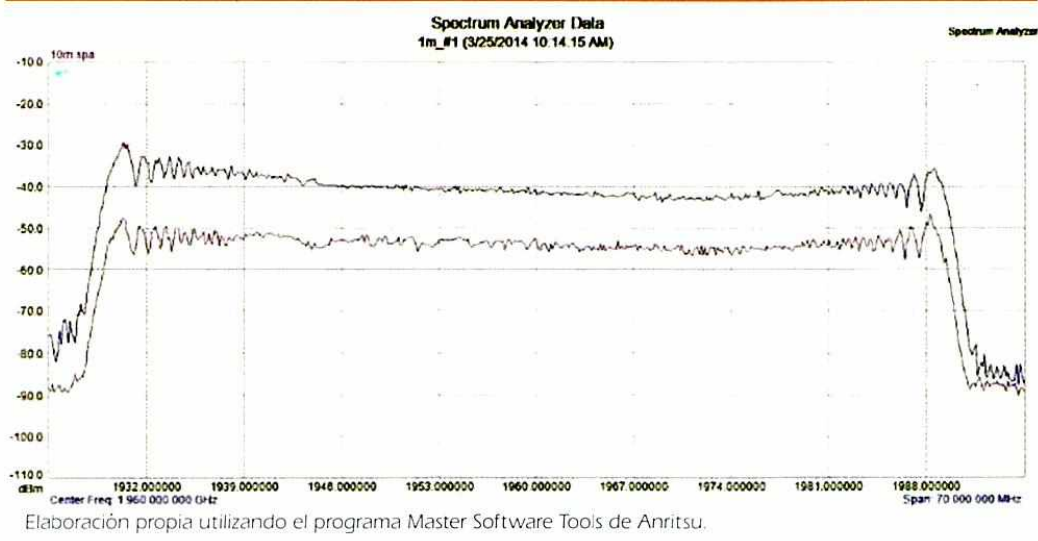

cada eje por separado (Narda Safety Test Solutions, 2007, pág. 1.3). Además, gracias a este tipo de sondas, las mediciones son independientes de la polarización y dirección del emisor (Miclaus \& Bechet, 2007, pág. 6). El equipo, junto a la sonda de medición, se muestra en la Figura 12.
Para realizar mediciones actuales y de manera continua obteniendo un valor de intensidad de campo aproximadamente cada segundo, el equipo fue gestionado $y$ configurado por medio del software SRM-TS de Narda Safety Test Solutions, instalado en un computador portátil Lenovo T61, mostrado en la Figura 12. Adicionalmente, se puede observar que la sonda está colocada en un trípode no conductivo marca Berlebach y que está conectada al medidor por medio de un cable coaxial de 1.5 metros de longitud, el cual, de acuerdo a documentación del fabricante, no ocasiona ningún efecto sobre los resultados de la medición (Narda Safety Test Solutions, 2007).

A continuación, en la Tabla 2 se observa los parámetros de configuración del equipo Narda SRM-3000 que se usaron para realizar las mediciones de intensidad de campo eléctrico.

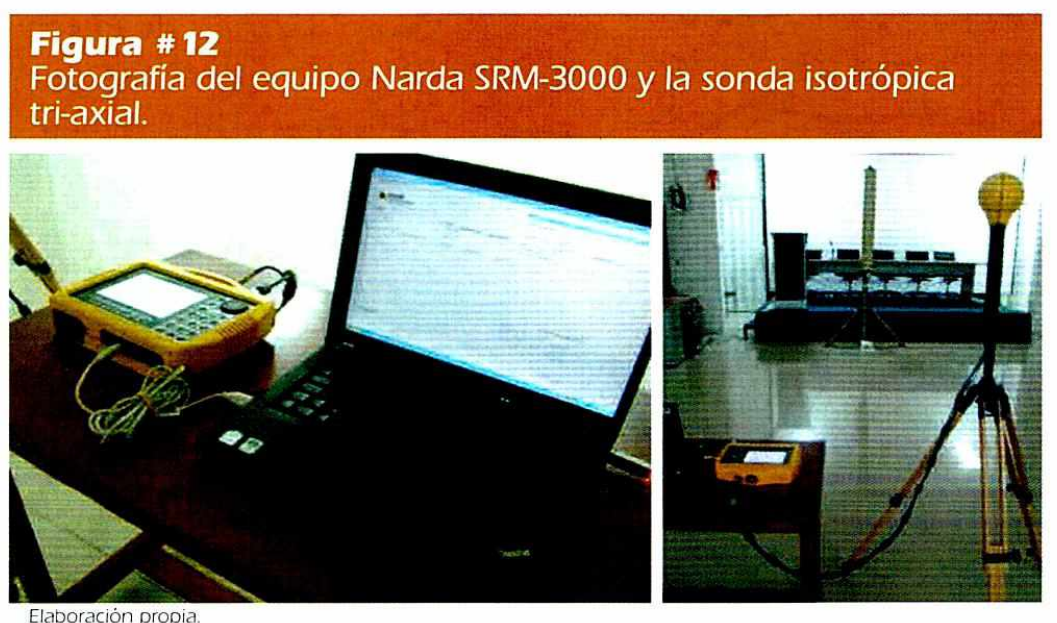

61 


\section{Tabla \# 2}

Parámetros de configuración del equipo Narda de campo eléctrico

\begin{tabular}{ll}
\hline Modo de operación & Safety Evaluation \\
\hline Tabla de servicio & OTECEL 1900 \\
\hline Frecuencia minima & $1945 \mathrm{MHz}$ \\
\hline Frecuencia máxima & $1950 \mathrm{MHz}$ \\
\hline RBW (Resolution bandwidth) & $1 \mathrm{MHz}$ (Auto) \\
\hline Rango de medición (MR) & $4 \mathrm{~V} / \mathrm{m}$ \\
\hline Tipo de resultado & $\mathrm{ACT}$ (mediciones actuales) \\
\hline Unidad de medición & $\mathrm{V} / \mathrm{m}$ \\
\hline Nombre de cable & SRM 1.5m \\
\hline Nombre de antena & $3 \mathrm{AX} 75 \mathrm{M}-3 \mathrm{G}$ \\
\hline Tipo de medición & Isotropic Result (resultados isotrópicos) \\
\hline Fuente: Elaboración propia & \\
\hline
\end{tabular}

Luego de la configuración del equipo y preparación del área de medición, se procedió a realizar mediciones de intensidad de campo eléctrico en los diez puntos de medición establecidos sin una fuente generadora de señales, es decir, sin utilizar el equipo inhibidor. El objetivo de esta prueba es comprobar que sin la influencia de fuentes externas e internas significativas en los puntos de medición los valores de intensidad de campo eléctrico deben permanecer prácticamente constantes, sin importar la ubicación del punto. En la Figura 13 se observan los resultados de estas mediciones, indicando los valores máximos, mínimos y los promedios de cada punto, considerando mediciones durante 30 minutos en cada uno de ellos. SRM-3000 para realizar las mediciones de intensidad

Como se pudo observar en la Figura 13, los valores de intensidad de campo eléctrico a lo largo de los diez metros se mantienen constantes, con valores promedio de alrededor de $0.006 \mathrm{~V} / \mathrm{m}$, valores mínimos de $0.004 \mathrm{~V} / \mathrm{m}$ y máximos de $0.008 \mathrm{~V} / \mathrm{m}$, considerando el número total de muestras en cada punto, por lo que se deduce que no hay presencia significativa de señales electromagnéticas en la banda medida.

Mientras que las primeras pruebas constituían una fase previa y complementaria para este análisis, el principal enfoque del presente estudio es comprobar que los niveles de intensidad de campo eléctrico disminuyen conforme aumenta la distancia entre el receptor y la fuente. Estas mediciones fueron realizadas utilizando los mismos equipos que en las pruebas anteriores, adicionando el inhibidor para generar una señal semejante en frecuencia a las señales de telefonía móvil. Las mediciones fueron realizadas en los diez puntos establecidos con una separación de un metro, colocando la

Figura \# 13

Gráfico de los valores promedio, mínimo y máximo de los diez puntos de medición.

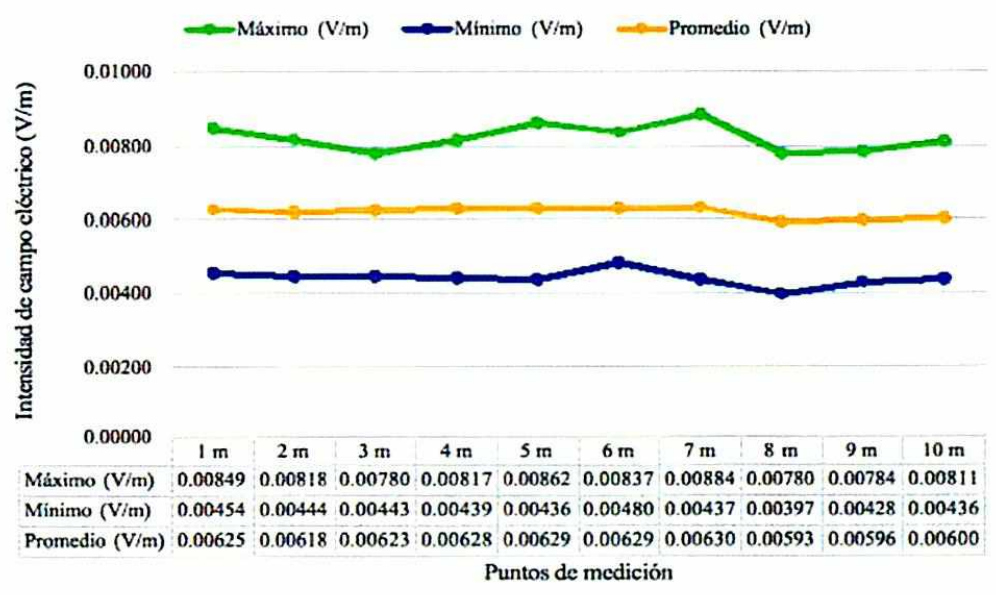
sonda a una altura de 1.5 m. El Narda SRM-3000 permitió capturar los valores actuales de intensidad de campo eléctrico durante treinta minutos, obteniendo aproximadamente un valor cada segundo. En las Figuras 14 y 15 se observa el área donde se realizaron las mediciones y la distribución de los equipos. 
Figura \# 14

Fotografía del área donde se realizaron las mediciones de intensidad de campo eléctrico.

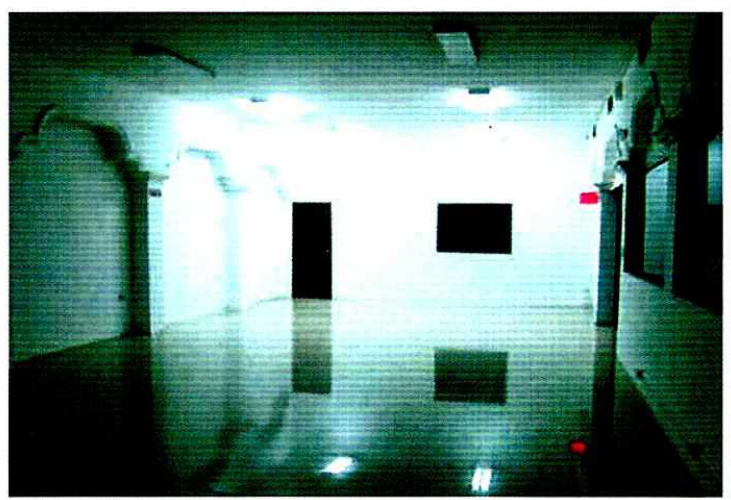

Elaboración propia.

\section{Figura \# 15}

Ubicación de la antena celular, medidor de radiación, computador portátil y sonda de medición.

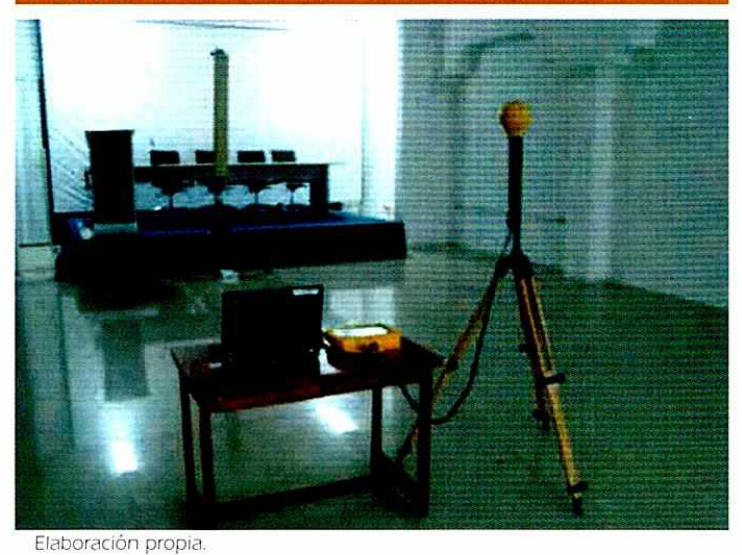

Elaboración propia

A partir de estas mediciones realizadas a diferentes distancias a partir de la fuente, se obtuvieron los resultados que se encuentran en la siguiente sección.

\section{Análisis de los re- sultados}

Luego de realizar las mediciones en los diez puntos establecidos durante treinta minutos en cada uno de ellos, se procedió a descargar la información y procesarla. La información relevante resultante de estas mediciones incluyen datos como: fecha y hora de medición, frecuencia mínima, frecuencia máxima e intensidad de campo eléctrico.

Al ser la intensidad de campo eléctrico una variable cuantitativa continua, es necesario agrupar los valores resultantes de las mediciones en los diez puntos y presentarlos en tablas de distribución de frecuencias y, a partir de los intervalos y frecuencias encontradas, elaborar histogramas de frecuencias.

A partir de los histogramas de frecuencias y del coeficiente de asimetría, se pudo observar que las distribuciones de las mediciones en los diez puntos son asimétricas negativas o sesgadas a la izquierda ya que los valores de la media aritmética son menores que los de la mediana y el coeficiente de asimetría tiene un valor negativo. Cuando se tiene este tipo de distribución, la medida de tendencia central más representativa es la mediana, el valor que divide la distribución de manera que el $50 \%$ de los valores estén por encima y el otro $50 \%$ por debajo de este valor. Esta medida usualmente va acompañada del rango como medida de dispersión. A continuación, en la Figura 16 se puede observar que los valores

\section{Figura \# 16 \\ Gráfico de los valores máximos, minimos y el rango de las} mediciones realizadas en los diez puntos.

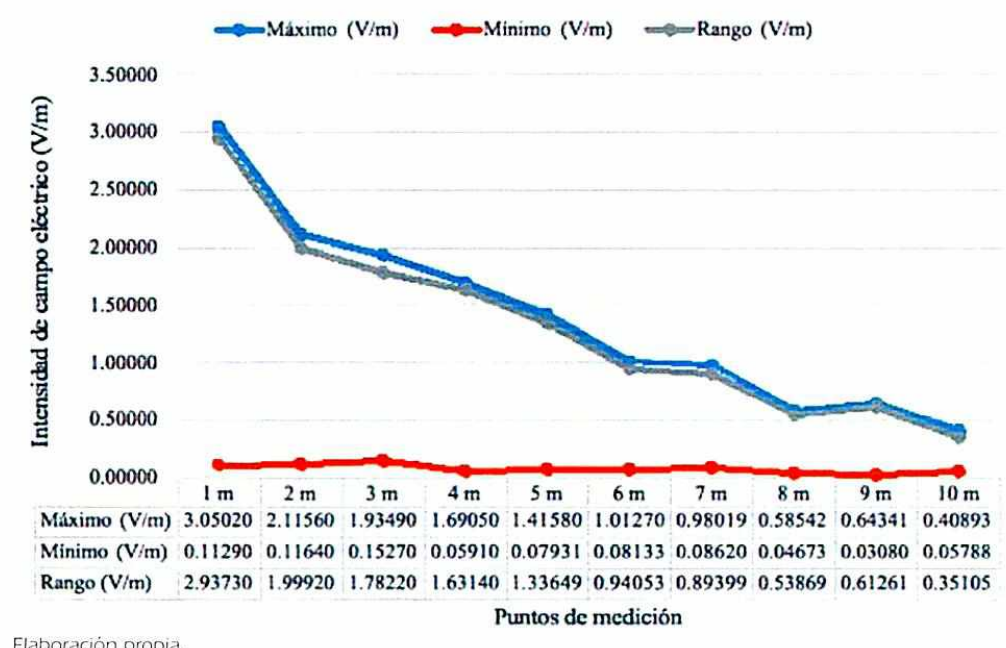

63

INVESTIGATIO No. 5, marzo 2014, pp 51-68 
del rango, es decir la diferencia entre el valor máximo y el valor mínimo, es comparable con el máximo, lo que puede interpretarse como una variabilidad significativa de los valores de intensidad de campo eléctrico medidos, la cual es mayor en los puntos más cercanos al transmisor.

La gran variabilidad de los valores de campo eléctrico medido puede deberse a tres factores: influencia de señales externas, variaciones en el equipo receptor o inestabilidad de la fuente. Luego de las mediciones realizadas sin la fuente generadora de señales se determinó que no existía influencia significativa de campos electromagnéticos externos ya que estos se mantenían casi constantes. Además, se determinó que el equipo receptor no ocasionaba gran variabilidad en los resultados ya que la diferencia entre los valores máximos y mínimos en cada uno de estos puntos no superaba $0.003 \mathrm{~V} / \mathrm{m}$.

A partir de lo expuesto se puede determinar que la variabilidad de los resultados está dada por la inestabilidad de la fuente, ya que los inhibidores de señal celular no son equipos diseñados para pruebas en laboratorio y presentan variaciones en la potencia de salida. En las Figuras 17 y 18 se puede observar la diferencia en la dispersión con respecto al promedio de las mediciones realizadas en el primer punto sin y con fuente generadora de señales, donde se evidencia que existe mayor variabilidad en las mediciones realizadas utilizando una fuente.

Como se mencionó anteriormente, la mediana es una medida de tendencia central representativa de las distribuciones asimétricas negativas. En la Figura 19 se observa un gráfico con la mediana de cada uno de los puntos de medición.

A partir de este gráfico se observa que la tendencia es que la intensidad de campo

\section{Figura \# 17}

Gráfico de dispersión de las mediciones

realizadas en el primer punto sin fuente

generadora de señales.

0.0025

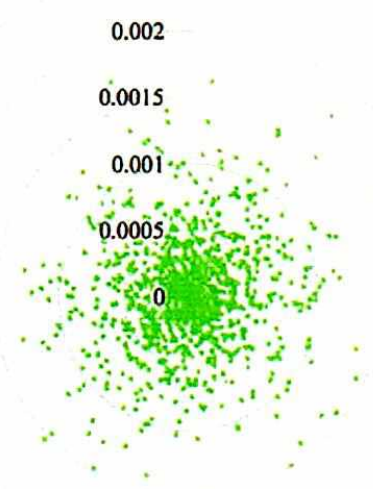

- Intensidad de campo eléctrico (V/m)

Elaboración propia.

\section{Figura \# 18}

Gráfico de dispersión de las mediciones

realizadas en el primer punto con fuente generadora de señales.

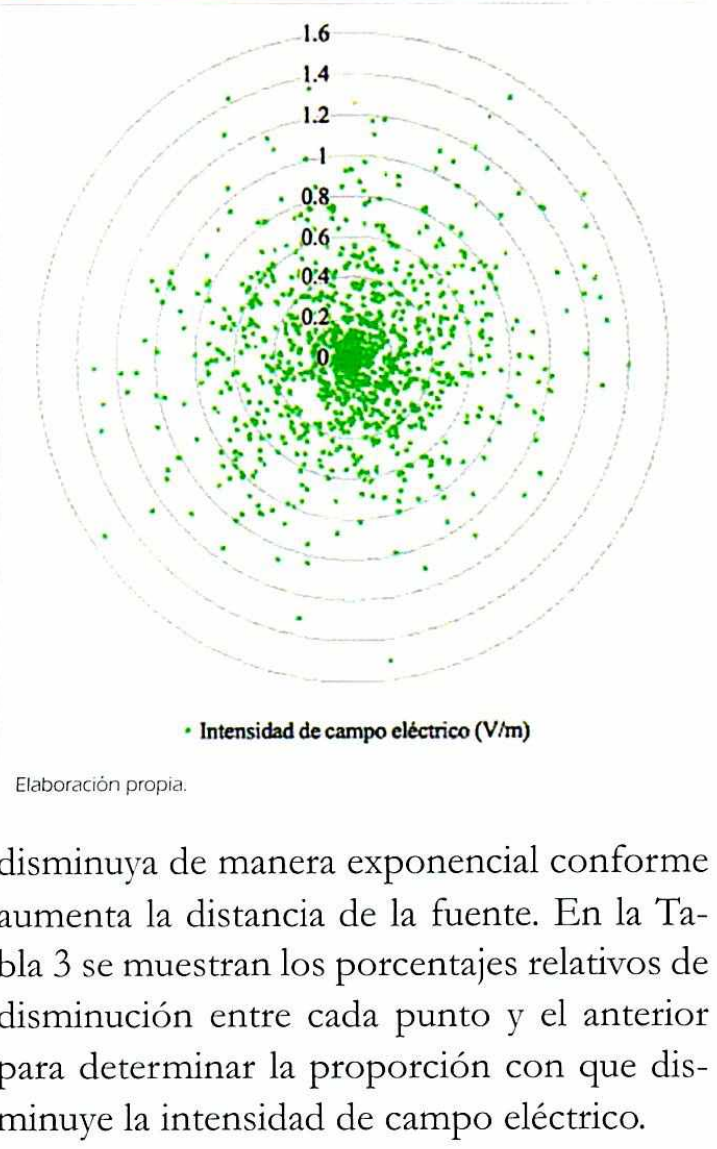


Figura \# 19

Gráfico de los valores de intensidad de campo eléctrico

medianos en cada punto de medición.

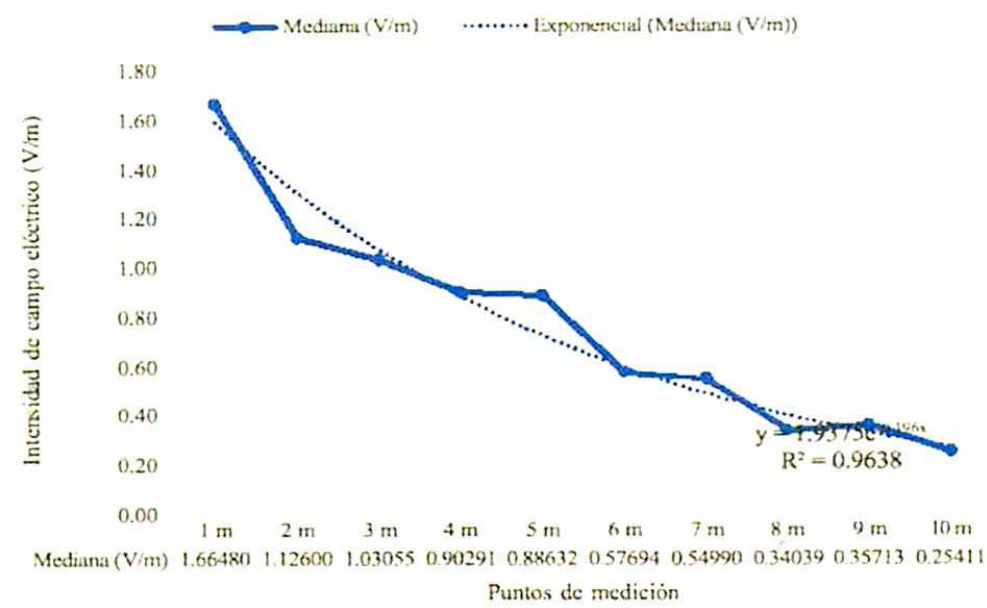

$y=$ ecuación de la linea de tendencia; $x$ = distancia de la fuente, $R 2$ - coeficiente de determinación Elaboración propia.

De estos resultados se concluye que la intensidad de campo eléctrico disminuve, pero no de manera uniforme conforme aumenta la distancia, sin embargo, la línea de tendencia, mostrada como una línea punteada en la Figura 19, tiene un coeficiente de determinación $\mathrm{R}^{2}$ igual a 0.9633 , el cual es muy cercano a uno lo que determina que los valores calculados para esta serie son muy próximos a los valores medianos de intensidad de campo eléctrico y podría usarse la ecuación exponencial $y=1.9375 \mathrm{e}^{-0.196 x}$ correspondientes a la línea de tendencia para encontrar porcentajes de disminución más acertados. Los diez valores calculados a partir de la línea de tendencia, así como los porcentajes relativos de disminución, se encuentran en la Tabla 4.

\section{Tabla \# 3}

Porcentaje de disminución de la intensidad de campo eléctrico respecto al punto anterior

\begin{tabular}{ccc}
$\begin{array}{c}\text { Punto de } \\
\text { medición }\end{array}$ & $\begin{array}{c}\text { Punto de } \\
\text { referencia }\end{array}$ & $\begin{array}{c}\text { Porcentaje de } \\
\text { disminucion } \\
2 \mathrm{~m}\end{array}$ \\
\hline $3 \mathrm{~m}$ & $2 \mathrm{~m}$ & $32.36 \%$ \\
\hline $4 \mathrm{~m}$ & $3 \mathrm{~m}$ & $8.48 \%$ \\
\hline $5 \mathrm{~m}$ & $4 \mathrm{~m}$ & $12.39 \%$ \\
\hline $6 \mathrm{~m}$ & $5 \mathrm{~m}$ & $1.84 \%$ \\
\hline $7 \mathrm{~m}$ & $6 \mathrm{~m}$ & $34.91 \%$ \\
\hline $8 \mathrm{~m}$ & $7 \mathrm{~m}$ & $4.69 \%$ \\
\hline $9 \mathrm{~m}$ & $8 \mathrm{~m}$ & $38.10 \%$ \\
\hline $10 \mathrm{~m}$ & $9 \mathrm{~m}$ & $-4.92 \%{ }^{\mathrm{a}}$ \\
\hline
\end{tabular}

Nota: Los porcentajes de disminución fueron calculados dividiendo la intensidad de campo eléctrico del punto de medicion para la del punto de referencia. Elaboración propia a Este porcentaje negativo significa que el valor de intensidad de campo eléctrico en el punto nueve fue 4.92 superior al del punto ocho.

\section{Tabla \#4}

\section{Valores de intensidad de campo eléctrico calculados a partir de la linea de tendencia y porcentajes de disminución respecto al punto anterior}

\begin{tabular}{cccc}
$\begin{array}{c}\text { Punto de } \\
\text { medición }\end{array}$ & $\begin{array}{c}\text { Intensidad campo } \\
\text { eléctrico }(\mathrm{V} / \mathrm{m})\end{array}$ & $\begin{array}{c}\text { Punto de } \\
\text { referencia }\end{array}$ & $\begin{array}{c}\text { Porcentaje de } \\
\text { disminución }\end{array}$ \\
\hline $1 \mathrm{~m}$ & 1.59265 &.- & - \\
\hline $2 \mathrm{~m}$ & 1.30918 & $1 \mathrm{~m}$ & $17.80 \%$ \\
\hline $3 \mathrm{~m}$ & 1.07616 & $2 \mathrm{~m}$ & $17.80 \%$ \\
\hline $4 \mathrm{~m}$ & 0.88462 & $3 \mathrm{~m}$ & $17.80 \%$ \\
\hline $5 \mathrm{~m}$ & 0.72717 & $4 \mathrm{~m}$ & $17.80 \%$ \\
\hline $6 \mathrm{~m}$ & 0.59774 & $5 \mathrm{~m}$ & $17.80 \%$ \\
\hline $7 \mathrm{~m}$ & 0.49135 & $6 \mathrm{~m}$ & $17.80 \%$ \\
\hline $8 \mathrm{~m}$ & 0.40389 & $7 \mathrm{~m}$ & $17.80 \%$ \\
\hline $9 \mathrm{~m}$ & 0.33201 & $8 \mathrm{~m}$ & $17.80 \%$ \\
\hline $10 \mathrm{~m}$ & 0.27291 & $9 \mathrm{~m}$ & $17.80 \%$ \\
\hline
\end{tabular}

Nota: Los porcentajes de disminución fueron calculados dividiendo la intensidad de campo eléctrico del punto de medición para la del punto de referencia. Elaboración propia 
$\Lambda$ partir de la Tabla 4 , se observa que al ser una función exponencial, los porcentajes de disminución son constantes a lo largo de los diez puntos de medición. Con esto se puede concluir que para un sistema con estas características, el nivel de intensidad de campo eléctrico disminuye aproximadamente un $17.80 \%$ cada metro que se aleja de la fuente.

\section{Conclusiones y recomendaciones}

Luego del análisis de presencia de campos electromagnéticas, se demostró que el sitio designado para la realización de las pruebas, no presenta señales portadoras en la banda D' de la operadora ( TTECEL S.A., que comprende el rango de frecuencias de $1945 \mathrm{MHz}$ a $1950 \mathrm{MHz}$, por lo que se puede asegurar que los resultados de las mediciones no fueron afectados de manera significativa por señales externas.

Al realizar las mediciones de la potencia de recepción, generada por el equipo inhibidor junto a la antena sectorial, se observó que estos niveles disminuyen conforme aumenta la distancia de la fuente, resultando en una diferencia de aproximadamente $12 \mathrm{dBm}$ entre el primer y el último punto.

En las mediciones de intensidad de campo eléctrico sin presencia de fuentes, es decir, con el equipo inhibidor apagado, se obtuvieron valores constantes en los die\% puntos de medición, variando entre $0.004 \mathrm{~V} / \mathrm{m}$ y 0.008 $\mathrm{V} / \mathrm{m}$, lo que significa que no existían señales externas significativas en la banda medida que puedan afectar los resultados de las mediciones.

Al efectuar las mediciones de intensidad de campo eléctrico de uno a diez metros de distancia respecto al inhibidor de señal celular, se observó que los rangos de cada uno de los diez puntos eran muy cercanos a los valores máximos, por lo que se concluye que los valores son muy dispersos entre ellos, con mayor grado en los puntos más cercanos a la fuente.

Luego de realizar el análisis del sitio y las mediciones de intensidad de campo eléctrico sin presencia de una fuente generadora de señales, se descartó la posibilidad de que la variabilidad de los resultados obtenidos utilizando el equipo inhibidor fuera ocasionada por señales externas o por la variabilidad del receptor. $\lambda$ partir de esto se concluye que esta dispersión está dada por la inestabilidad de la fuente ya que un inhibidor de señal celular no es un equipo ideado para obtener resultados completamente controlados dentro de un laboratorio.

Se concluye además que los valores medianos de cada uno de los puntos de medición disminuyen conforme aumenta la distancia de la fuente, tal como lo establece la teoría. Los valores tienen una tendencia a disminuir de manera exponencial de acuerdo a la forma $y=1.9375 \mathrm{e}-0.196 \mathrm{x}$. Considerando la línea de tendencia generada, el porcentaje de disminución de la intensidad de campo eléctrico es igual a $17.80 \%$ en cada metro.

Se recomienda realizar estas pruebas en un ambiente donde existan más variables controladas, como por ejemplo, utilizando un generador de señales en lugar de un equipo inhibidor o realizando las mediciones en un laboratorio o una cámara anecoica para garantizar que los resultados no se vean afectados por factores que no son controlados por el investigador.

$\Lambda$ dicional, se recomienda realizar un análisis para extrapolar estos resultados y comprobar si la ecuación obtenida es comparable con la establecida en las Recomendaciones y Reglamentos analizados.

Finalmente, se recomienda ejecutar un análisis detallado de las mediciones realiza- 
das en el sitio destinado para las pruebas, en cada uno de los rangos de frecuencias para identificar si existen otras bandas que sean esencialmente inmunes a señales electromagnéticas externas, y de ser el caso, poder adaptar el área para que cumpla las funciones de una cámara semi anecoica.

\section{Bibliografia}

American Cancer Society: (31 de enero de 2013). Cellular Pbone Tomers. Recuperado el 02 de abril de 2014, de American Cancer Society: http://www.cancer.org/ cancer/cancercauses/othercarcinogens/ athome/cellular-phone-towers

Biolnitiative Working Group. (2012). A Rationale for Biologically-based Exposure Standards for Lon-Intensity Electromagnetic Radiation. Cindy Sage and David O. Carpenter.

Comisión Internacional para la Protección de las Radiaciones no Ionizantes. (1998). Recomendaciones para limitar la exposición a campos eléctricos, magnéticos y electromagnéticos (basta 300 GHz). Health Physics, 79(4), 494-522.

Consejo Nacional de Telecomunicaciones. (2005). Reglamento de protección de emisiones de radiación no ionizante generadas por uso de frecuencias del espectro radioeléctrico. Quito.

GSMA. (12 de febrero de 2014). Comunicaciones móviles y Salud. Recuperado el 02 de abril de 2014, de GSMA: http://www. gsma.com/publicpolicy/mobile-communications-and-health

Health Protection Agency. (2012). Health Eiffects from Radiofrequency Eilectromagnetic Fields. London, United Kingdom.

Industry Canada. (2012). Case Study: Measurements of Radio Frequency Exposure from Wi-Fi Devices. Spectrum Management and
Telecommunications.

Jiménez, J. (2012). Propuesta de optimización de los paraimetros de calidad de servicio en base al análisis de los contratos de Servicio Móril Al'anzado de CONECHI S.A. y, OTECELS.A. Tesis, Escuela Politécnica Nacional, Iacultad de Ingeniería Eléctrica y Electrónica, Quito.

Lewicki, F. (25-26 de julio de 2011). L'se of EMF estimator for Base Station antborization in a multi-sonrce environment. ITU Workshop on "Practical measurement of ENFF exposure". Gaborone.

Niclaus, S., \& Bechet, P. (2007). Estimated and measured values of the radiofrequenc' radiation ponver density' around cellular base stations. Romanian Journal of Physics, 52(3-4), 429440.

Moreno, R., Güemes, J., \& Pico, I. (s.f.). Análisis del campo magnético en una linea eléctrica de AT. Universidad del País Vasco, Departamento de Ingeniería Eléctrica, Bilbao.

Narda Safety Test Solutions. (2007). SRM3000 Selective Radiation Meter Operating Mamual. 11/07.06, C. Pfullingen, Germany.

Organización Mundial de la Salud. (s.f.). Campos electromagnéticos (CEM) - ¿Qué son los campos electromagnéticos? Recuperado el 22 de marzo de 2014, de Organización Mundial de la Salud: http://wwwwwho. int/peh-emf/about/WhatisEMF/es/

Pogo, K. (2011). Análisis de los servicios de telecomunicaciones móviles en el Ecuador: Tesis, Escuela Politécnica del Ejército, Departamento de Eléctrica y Electrónica, Sangolquí.

Secretaría de Estado de Telecomunicaciones y para la Sociedad de la Información. 
(s.f.). Control del Espectro Radioeléctrico. Recuperado el 28 de marzo de 2014, de Ministerio de Industria, Energía y Turismo: http://www.minetur.gob.es/telecomunicaciones/Espectro/CTER/Paginas/ CTER.aspx

SENATEL, y OTECEL S.A. (2008). Contrato de concesión para la prestación de Servicio Móvil Aranzado, del Servicio Telefónico de Larga Distancia Internacional, los que podrán prestarse a través de terminales de telecomunicaciones de uso puiblico y concesión de las bandas de Frecuencias Es. Quito, Ecuador.

Skvarca, J., \& Aguirre, A. (2006). Normas y estándares aplicables a los campos electromagnéticos de radio frecuencias en América Latina - guía para los límites de exposición y los protocolos de medición. Rerista Panamericana de Salud Priblica, 20(2/3), 205-212.

Unión Internacional de Telecomunicaciones. (2000). Recomendación UTT-T K.52: Orientación sobre el cumplimiento de los limites de exposición de las personas a los campos electromangéticos.

Unión Internacional de Telecomunicaciones. (2003). Recomendación UIT-T K.61: Directrices sobre la medición y la predicción numérica de los campos electromagnéticos para comprobar que las instalaciones de telecomunicaciones.

Unión Internacional de Telecomunicaciones. (2011). Recomendación UIT-T K.83: Supervisión de los niveles de intensidad del campo electromagnético.

\section{Nataly Idrovo López}

Ing. en Telecomunicaciones de la Universidad Espíritu Santo - UEES

E-mail: nidrovo@uees.edu.ec

\section{Franklin Chenche Villacis}

Ing. en electricidad especialización en electrónica de la Escuela Superior Politécnica Del Litoral (ESPOL)

Magister en Gestión de proyectos

Universidad Espíritu Santo - Ecuador

E-mail: frchenche@uees.edu.ec 